\title{
¿Cómo optimizar los recursos en México para el tratamiento oncológico en la era de la inmunoterapia?
}

\author{
Eleazar Omar Macedo-Pérez* \\ Unidad Funcional de Tumores Torácicos, Instituto Nacional de Cancerología, Ciudad de México, México
}

En la actualidad, las opciones de tratamiento sistémico contra el cáncer incluyen quimioterapia, terapias blanco e inmunoterapias. Avances importantes en este tipo de tratamientos han llevado a una mejoría sustancial en la supervivencia global y en el porcentaje de pacientes vivos a largo plazo, sobre todo con el empleo de las modernas inmunoterapias (inhibidores de checkpoints inmunológicos que incluyen terapias anti Cytotoxic T-Lymphocyte Antigen 4 [anti-CTL-4], anti Programmed Death Ligand 1 [anti-PD-1] y anti Programmed Death Ligand 1 [anti-PD-L1]). La primera terapia anti-PD-1 aprobada fue el nivolumab, que demostró beneficio clínico en pacientes con varios tipos de tumores que habían recibido varias líneas de tratamiento previamente (cáncer de pulmón de células no pequeñas [CPCNP] avanzado, melanoma metastásico, cáncer renal metastásico, cáncer de próstata metastásico resistente a castración y cáncer colorrectal metastásico). La dosis aprobada en el estudio fase I fue de $3 \mathrm{mg} / \mathrm{kg}$ de peso administrada cada 2 semanas ${ }^{1}$. En dos estudios subsecuentes fase III (CheckMate $017^{2}$ y CheckMate $057^{3}$ ) que llevaron a la aprobación del nivolumab en segunda línea en pacientes con CPCNP, la dosis empleada fue de $3 \mathrm{mg} / \mathrm{kg}$ cada 2 semanas, que comparado con el docetaxel mostró beneficio en supervivencia global, en tasas de respuesta y un mejor perfil de seguridad ${ }^{2,3}$. Recientemente, con los argumentos de facilitar el empleo del nivolumab, en cuanto a la dosis y tiempo de preparación del medicamento, se realizó un análisis de su perfil clínico y farmacológico en 1,895 pacientes que participaron en 11 diferentes ensayos clínicos de esta molécula, en donde recibieron dosis de $0.3-10 \mathrm{mg} / \mathrm{kg}$ cada 2 semanas (Fig. 1) ${ }^{4}$. La conclusión obtenida es que no hubo diferencias entre la dosis de $3 \mathrm{mg} / \mathrm{kg}$ y la dosis de $10 \mathrm{mg} / \mathrm{kg}$ administrados cada 2 semanas, considerando que el empleo de una dosis fija, intermedia entre estas 2 dosis, podría ser adecuada en pacientes candidatos a recibir este tratamiento.

Se realizó un estudio para seleccionar la dosis fija (flat doses) de nivolumab, tomando en cuenta el peso corporal de 3,458 pacientes enrolados en 18 ensayos clínicos de nivolumab de múltiples neoplasias, incluyendo melanoma, CPCNP, cáncer renal, cáncer pulmonar de células pequeñas, linfoma no Hodgkin y tumores de cabeza y cuello, entre otros. La mediana de peso en la población estudiada fue de $77 \mathrm{~kg}$, por lo que se determinó calcular la dosis basada en un peso promedio de $80 \mathrm{~kg}$, de manera, que la dosis fija resultante de multiplicar $3 \mathrm{mg} / \mathrm{kg}$ por $80 \mathrm{~kg}$ fue de $240 \mathrm{mg}$ cada 2 semanas. Entre la dosis fija de $240 \mathrm{mg}$ y la dosis por $\mathrm{kg} /$ peso administradas cada 2 semanas no hubo diferencias en las concentraciones y eficacia entre ambos esquemas de tratamiento (Fig. $2 \mathrm{~A}$ y B $)^{5}$.

Posteriormente a este estudio, los ensayos clínicos realizados han empleado la dosis fija de $240 \mathrm{mg}$ cada 2 semanas y esta ha sido aprobada por la Food and Drug Administration (FDA) y la Entidad Mexicana de

\section{Correspondencia:}

*Eleazar Omar Macedo-Pérez Instituto Nacional de Cancerología

Unidad Funcional de Tumores torácicos

Av. San Fernando, 22

Col. Sección XVI, Del. Tlalpan

Fecha de recepción: 04-04-2019

Fecha de aceptación: 15-04-2019

DOI: 10.24875/j.gamo.M19000175
Disponible en internet: 12-06-2019 Gac Mex Oncol. 2019;18:42-47 www.gamo-smeo.com 


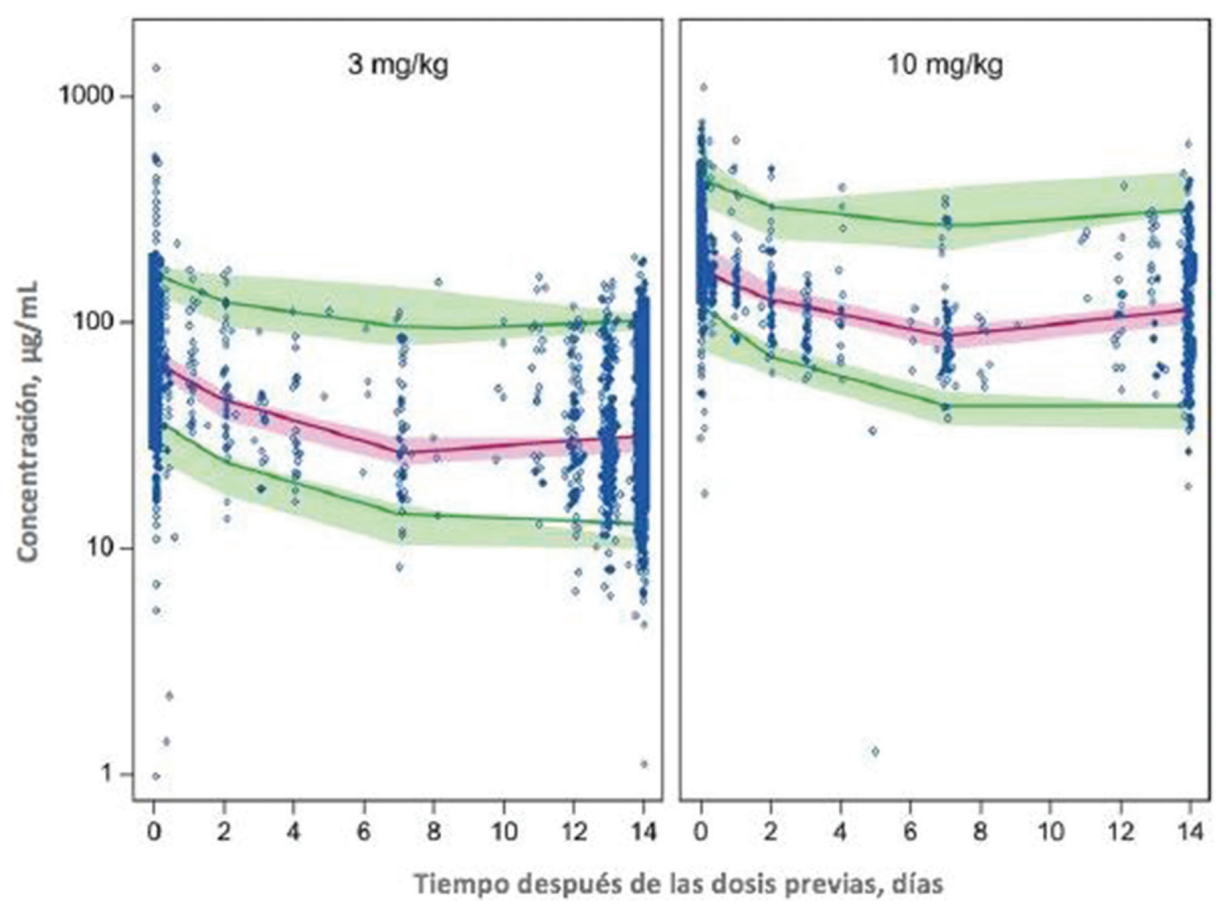

Figura 1. Se observan similares concentraciones de nivolumab entre las dos dosis (3 mg/kg vs. $10 \mathrm{mg} / \mathrm{kg}$ ) (adaptada de Bajaj, et al., 20174).

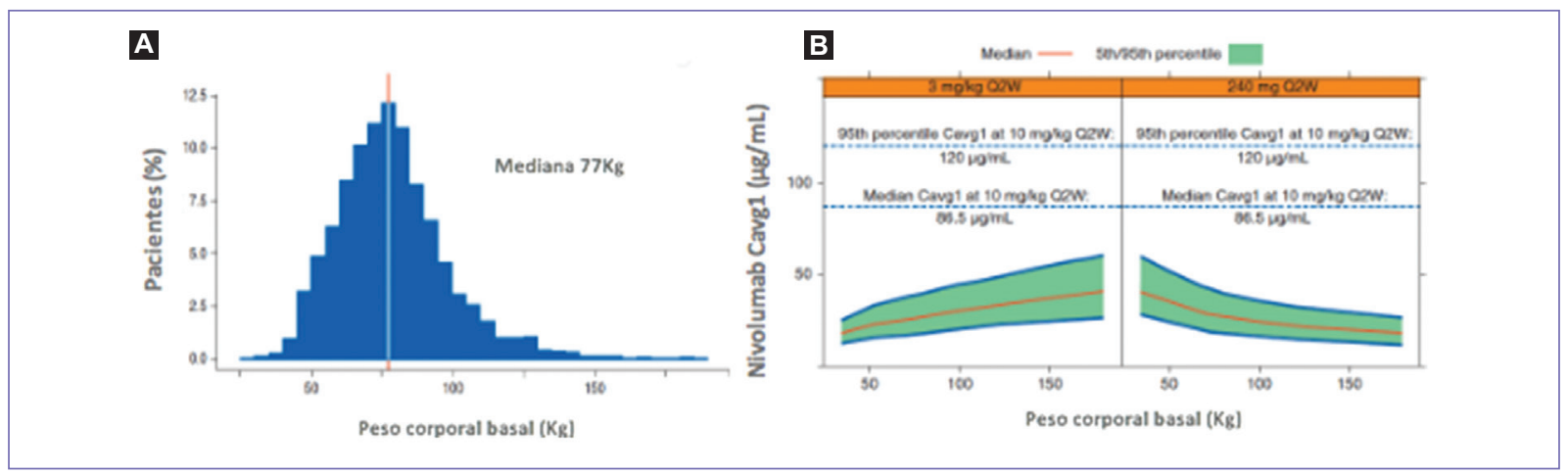

Figura 2. La mediana del peso en la población analizada fue de $77 \mathrm{~kg}$ (A). Comparando la dosis de $3 \mathrm{mg} / \mathrm{kg}$ de peso vs. la dosis fija de $240 \mathrm{mg}$ administrados cada 2 semanas (B) no hay diferencias en concentraciones y proporción de efectos adversos y parámetros de eficacia y seguridad (adaptada de Zhao, et al., 20175).

Acreditación (EMA). Existe la presentación de nivolu$\mathrm{mab}$ de $100 \mathrm{mg} / 10 \mathrm{ml}$ y $40 \mathrm{mg} / 4 \mathrm{ml}$.

Otra terapia anti-PD-1 aprobada en diversas neoplasias es el pembrolizumab. En un estudio fase I típico de diseño $3 \times 3$ con escalamiento de dosis, en el cual fueron incluidos 32 pacientes, una dosis $\geq 2 \mathrm{mg} / \mathrm{kg}$ administrada i.v. cada 3 semanas fue segura y presentó actividad clínica ${ }^{6}$. Basados en este primer estudio, se realizó un estudio fase I adaptativo, robusto, que incluyó a 1,235 pacientes (655 pacientes con melanoma y 550 pacientes con CPCNP), en el que se probaron diversas hipótesis, incluyendo análisis de eficacia por líneas de Tx previas, expresión de PD-L1 vs. tumores PD-L1 negativos, así como dos distintas dosis: $2 \mathrm{mg} / \mathrm{kg}$ administradas cada 2 o cada 3 semanas y $10 \mathrm{mg} / \mathrm{kg}$ administradas cada 2 y 3 semanas. Con este estudio se consiguieron 2 aprobaciones: para el CPCNP con expresión de PD-L1 > 50\% en pacientes previamente 


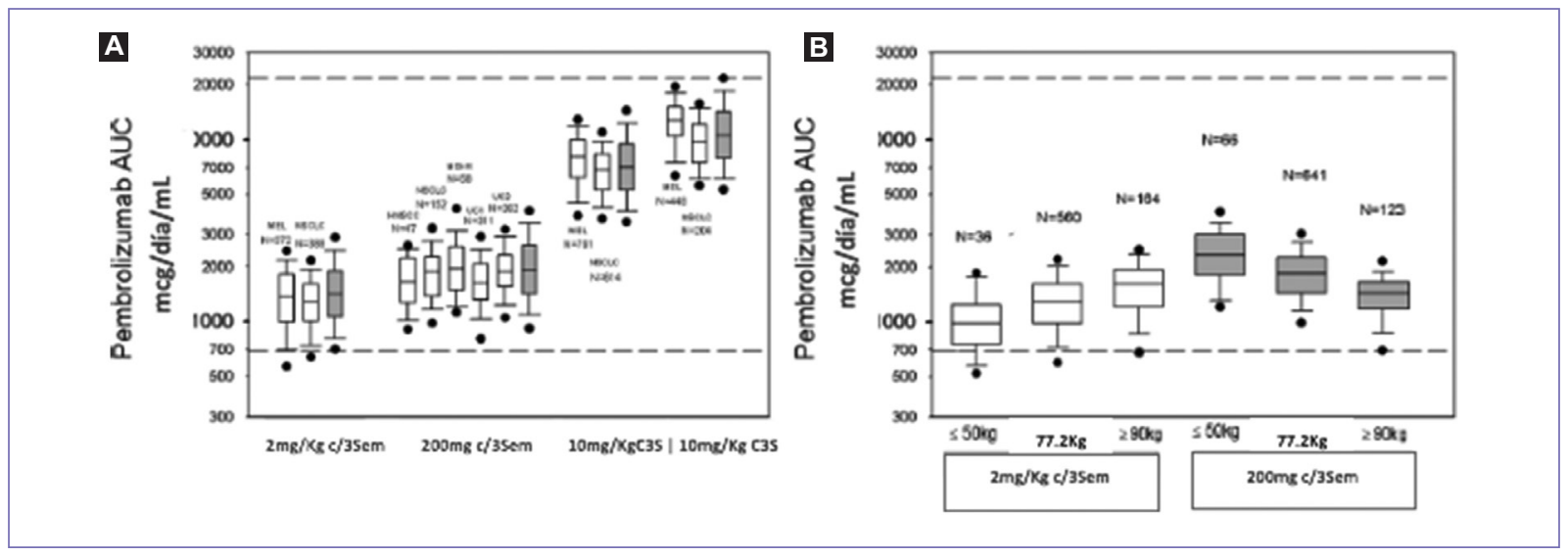

Figura 3. A la izquierda se observan las concentraciones de pembrolizumab (AUC) con diferentes dosis. Se pude notar que no hay diferencias significativas entre $2 \mathrm{mg} / \mathrm{kg}$ cada 3 semanas (q3w) vs $200 \mathrm{mg}$ Q3W. A la derecha, se observan las concentraciones de pembrolizumab de acuerdo al peso corporal: hay un exceso de dosis (con el esquema de dosis fija) en pacientes con peso corporal $<50 \mathrm{~kg}$ vs dosis por kg/peso. (adaptada de Freshwater, et al., 2017 ${ }^{10}$ ).

Tabla 1. Análisis del peso de 792 pacientes del Instituto Nacional de Cancerología, candidatos a recibir inmunoterapia para el tratamiento del cáncer de pulmón de células no pequeñas. En el recuadro rojo se muestra al gran número de pacientes que recibirían un exceso de dosis de pembrolizumab y nivolumab, respectivamente. El exceso de gasto por paciente por cada aplicación de nivolumab (cada 2 semanas) sería de $\$ 15,390.00$ y para el pembrolizumab de $\$ 52,500.00$ por aplicación cada 3 semanas, empleando las dosis fijas aprobadas para cada tratamiento

\begin{tabular}{|c|c|c|c|c|c|c|c|}
\hline Peso & Peso (kg) & $\begin{array}{c}\text { Dosis/kg } \\
\text { pembrolizumab } \\
\text { (promedio) }\end{array}$ & $\begin{array}{l}\text { Exceso de dosis } \\
\text { pembrolizumab }\end{array}$ & $\begin{array}{c}\text { Dosis/kg } \\
\text { nivolumab } \\
\text { (promedio) }\end{array}$ & $\begin{array}{l}\text { Exceso de } \\
\text { dosis } \\
\text { nivolumab }\end{array}$ & $\begin{array}{c}\text { Excesos de } \\
\text { costos } \\
\text { pembrolizumab* }\end{array}$ & $\begin{array}{l}\text { Exceso de } \\
\text { costos de } \\
\text { nivolumab }\end{array}$ \\
\hline Mínimo & 34.5 & $69 \mathrm{mg}$ & +131 mg & $103.5 \mathrm{mg}$ & $+136.5 \mathrm{mg}$ & $+\$ 98,250.00$ & $+\$ 46,683.00$ \\
\hline Máximo & 130.8 & $261.6 \mathrm{mg}$ & $-61.6 \mathrm{mg}$ & $392.4 \mathrm{mg}$ & $-152.4 \mathrm{mg}$ & $-46,200.00$ & $-\$ 52,150.80$ \\
\hline $\begin{array}{l}\text { Mediana } \\
\text { (DE) }\end{array}$ & $\begin{array}{c}65 \\
13.11\end{array}$ & $\begin{array}{c}130 \mathrm{mg} \\
( \pm 39 \mathrm{mg})\end{array}$ & $70 \mathrm{mg}$ & $195 \mathrm{mg}$ & $45 \mathrm{mg}$ & $+\$ 52,500.00$ & $\$ 15,390.00$ \\
\hline$\leq 50 \mathrm{~kg}$ & $\begin{array}{l}100 / 792 \\
(12.5 \%)\end{array}$ & $\begin{array}{l}<100 \mathrm{mg} \\
(100 \mathrm{mg})\end{array}$ & $100 \mathrm{mg}$ & $150 \mathrm{mg}$ & $90 \mathrm{mg}$ & $+\$ 75,000.00$ & $\$ 30,780.00$ \\
\hline$\geq 51-60 \mathrm{~kg}$ & $\begin{array}{l}199 / 792 \\
(25.1 \%)\end{array}$ & $\begin{array}{l}102-120 \mathrm{mg} \\
(110 \mathrm{mg})\end{array}$ & $90 \mathrm{mg}$ & $165 \mathrm{mg}$ & $75 \mathrm{mg}$ & $+\$ 67,500.00$ & $\$ 25,650.00$ \\
\hline$\geq 61-70 \mathrm{~kg}$ & $\begin{array}{l}251 / 792 \\
(31.7 \%)\end{array}$ & $\begin{array}{l}122-140 \mathrm{mg} \\
(130 \mathrm{mg})\end{array}$ & $70 \mathrm{mg}$ & $195 \mathrm{mg}$ & $45 \mathrm{mg}$ & $+\$ 52,500.00$ & $\$ 15,390.00$ \\
\hline$\geq 71-80 \mathrm{~kg}$ & $\begin{array}{c}150 / 792 \\
(19 \%)\end{array}$ & $\begin{array}{l}142-160 \mathrm{mg} \\
(150 \mathrm{mg})\end{array}$ & $50 \mathrm{mg}$ & $225 \mathrm{mg}$ & $15 \mathrm{mg}$ & $+\$ 37,500.00$ & $\$ 5,130.00$ \\
\hline$\geq 81-90 \mathrm{~kg}$ & $\begin{array}{l}60 / 792 \\
(7.6 \%)\end{array}$ & $\begin{array}{l}162-180 \mathrm{mg} \\
(170 \mathrm{mg})\end{array}$ & $30 \mathrm{mg}$ & $255 \mathrm{mg}$ & $-15 \mathrm{mg}$ & $+\$ 22,500.00$ & $-\$ 5,130.00$ \\
\hline$\geq 91-100 \mathrm{~kg}$ & $\begin{array}{c}24 / 792 \\
(3 \%)\end{array}$ & $\begin{array}{l}182-200 \mathrm{mg} \\
(190 \mathrm{mg})\end{array}$ & $10 \mathrm{mg}$ & $285 \mathrm{mg}$ & $-45 \mathrm{mg}$ & $\$ 7,500.00$ & $-\$ 15,390.00$ \\
\hline$\geq 101 \mathrm{~kg}$ & $\begin{array}{l}8 / 792 \\
(1 \%)\end{array}$ & (> $200 \mathrm{mg})$ & $-20 \mathrm{mg}$ & $303 \mathrm{mg}$ & $-90 \mathrm{mg}$ & $-15,000.00$ & $-\$ 30.780 .00$ \\
\hline
\end{tabular}

La dosis fija de pembrolizumab es de $200 \mathrm{mg}$ i.v. cada 3 semanas vs. $2 \mathrm{mg} / \mathrm{kg}$ de peso cada 3 semanas. La dosis fija de nivolumab es de $240 \mathrm{mg}$ i.v. cada 2 semanas vs. $3 \mathrm{mg} / \mathrm{kg}$ de peso cada 2 semanas. Duración del tratamiento con nivolumab: hasta progresión de la enfermedad o toxicidad inaceptable. Duración del tratamiento: hasta progresión de la enfermedad, toxicidad inaceptable 0 alcanzar 2 años. Costo por mg de pembrolizumab: $750 \mathrm{mg}$ (cada frasco de $100 \mathrm{mg}$ con un costo de $\$ 75,000.00$ ). Costo por mg de nivolumab: $\$ 342.00$ (cada frasco de $100 \mathrm{mg}$ con un costo de $\$ 34,200.00$ ) * Por aplicación de pembrolizumab de cada paciente.

†Por aplicación de pembrolizumab de cada paciente. 
Tabla 2. Exceso de costos del tratamiento del cáncer de pulmón de células no pequeñas (CPCNP) con pembrolizumab por 1 año de tratamiento con esquema de dosis fija

\begin{tabular}{|c|c|c|c|c|c|c|c|}
\hline Peso & Peso (kg) & $\begin{array}{c}\text { Dosis/kg } \\
\text { pembrolizumab } \\
\text { (promedio) }\end{array}$ & $\begin{array}{c}\text { Exceso de } \\
\text { dosis } \\
\text { pembrolizumab }\end{array}$ & $\begin{array}{c}\text { Excesos de } \\
\text { costos } \\
\text { pembrolizumab }\end{array}$ & $\begin{array}{l}\text { Costo anual } \\
\text { pembrolizumab } \\
\text { dosis fija }\end{array}$ & $\begin{array}{l}\text { Costo anual } \\
\text { pembrolizumab } \\
\text { dosis/kg }\end{array}$ & $\begin{array}{l}\text { Exceso de } \\
\text { gasto } \\
\text { anual por } \\
\text { paciente }\end{array}$ \\
\hline Mínimo & 34.5 & $69 \mathrm{mg}$ & $+131 \mathrm{mg}$ & $+\$ 98,250$ & $\$ 2,550,000$ & $\$ 879.750$ & $+\$ 1,670,250$ \\
\hline Máximo & 130.8 & $261.6 \mathrm{mg}$ & $-61.6 \mathrm{mg}$ & $-46,200$ & $\$ 2,550,000$ & $\$ 3,335,400$ & $-\$ 785,400$ \\
\hline $\begin{array}{l}\text { Mediana } \\
\text { (DE) }\end{array}$ & $\begin{array}{c}65 \\
13.11\end{array}$ & $\begin{array}{c}130 \mathrm{mg} \\
( \pm 39 \mathrm{mg})\end{array}$ & $70 \mathrm{mg}$ & $+\$ 52,500$ & $\$ 2,550,000$ & $\$ 1,657.500$ & $\$ 892,500$ \\
\hline$\leq 50 \mathrm{~kg}$ & $\begin{array}{l}100 / 792 \\
(12.5 \%)\end{array}$ & $\begin{array}{l}<100 \mathrm{mg} \\
(100 \mathrm{mg})\end{array}$ & $100 \mathrm{mg}$ & $+\$ 75,000$ & $\$ 2,550,000$ & $\$ 1,225.000$ & $\$ 1,275,000$ \\
\hline$\geq 51-60 \mathrm{~kg}$ & $\begin{array}{l}199 / 792 \\
(25.1 \%)\end{array}$ & $\begin{array}{c}102-120 \mathrm{mg} \\
(110 \mathrm{mg})\end{array}$ & $90 \mathrm{mg}$ & $+\$ 67,500$ & $\$ 2,550,000$ & $\$ 1,402.500$ & $\$ 1,147,500$ \\
\hline$\geq 61-70 \mathrm{~kg}$ & $\begin{array}{l}251 / 792 \\
(31.7 \%)\end{array}$ & $\begin{array}{c}122-140 \mathrm{mg} \\
(130 \mathrm{mg})\end{array}$ & $70 \mathrm{mg}$ & $+\$ 52,500$ & $\$ 2,550,000$ & $\$ 1,657.500$ & $\$ 892,500$ \\
\hline$\geq 71-80 \mathrm{~kg}$ & $\begin{array}{c}150 / 792 \\
(19 \%)\end{array}$ & $\begin{array}{c}142-160 \mathrm{mg} \\
(150 \mathrm{mg})\end{array}$ & $50 \mathrm{mg}$ & $+\$ 37,500$ & $\$ 2,550,000$ & $\$ 1,912,500$ & $\$ 637,500$ \\
\hline$\geq 81-90 \mathrm{~kg}$ & $\begin{array}{l}60 / 792 \\
(7.6 \%)\end{array}$ & $\begin{array}{c}162-180 \mathrm{mg} \\
(170 \mathrm{mg})\end{array}$ & $30 \mathrm{mg}$ & $+\$ 22,500$ & $\$ 2,550,000$ & $\$ 2,167,500$ & $\$ 382,500$ \\
\hline$\geq 91-100 \mathrm{~kg}$ & $\begin{array}{c}24 / 792 \\
(3 \%)\end{array}$ & $\begin{array}{c}182-200 \mathrm{mg} \\
(190 \mathrm{mg})\end{array}$ & $10 \mathrm{mg}$ & $\$ 7,500$ & $\$ 2,550,000$ & $\$ 2,422,500$ & $\$ 127,500$ \\
\hline$\geq 101 \mathrm{~kg}$ & $\begin{array}{l}8 / 792 \\
(1 \%)\end{array}$ & (> $200 \mathrm{mg})$ & $-20 \mathrm{mg}$ & $-15,000$ & $\$ 2,550,000$ & $\$ 2,805.000$ & $\$ 255,000$ \\
\hline
\end{tabular}

Indicaciones del pembrolizumab:

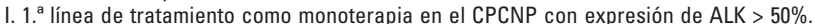

II. 1. ${ }^{\text {a }}$ línea de tratamiento en CPCNP en combinación con quimioterapia (cualquier valor de PD-L1).

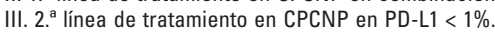

Resultados en los estudios pivotales:

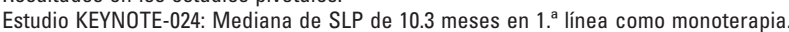

Estudio KEYNOTE-189: Mediana de SLP 8.8 meses en $1 .^{\text {a }}$ línea con quimioterapia.

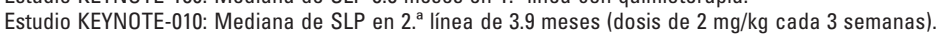

Costo de $200 \mathrm{mg}$ pembrolizumab cada 3 semanas $=\$ 150,000.00$

PD-L1: Proteínas de puntos de control, como PD-L1 (en las células tumorales); SLP: Síndrome linfoproliferativo

tratados y en melanoma metastásico en segunda línea, así como el empleo de una prueba como companion diagnostic en $\mathrm{CPCNP}^{7}$. En un análisis de los pacientes con diagnóstico de CPCNP del estudio KEYNOTE-001 no se encontraron diferencias en tasas de respuesta ni en eficacia entre las dosis de $2-10 \mathrm{mg} / \mathrm{kg}$ cada 3 semanas, apoyando el empleo de la dosis de $2 \mathrm{mg} / \mathrm{kg}$ cada 3 semanas $^{8}$. Finalmente, se realizó un estudio fase III con 3 brazos de tratamiento en pacientes con diagnóstico de CPCNP previamente tratados, con expresión de PD-L1 $\geq 1$ comparando pembrolizumab a dosis de $2 \mathrm{mg} / \mathrm{kg}$ cada 3 semanas y pembrolizumab $10 \mathrm{mg} / \mathrm{kg}$ cada 3 semanas vs. docetaxel $75 \mathrm{mg} / \mathrm{kg}$ cada 3 semanas. El pembrolizumab demostró ventajas sobre el docetaxel en supervivencia global, tasas de respuesta y perfil de seguridad. No hubo diferencias entre las dosis de pembrolizumab, lo que apoya el empleo de la dosis baja de $2 \mathrm{mg} / \mathrm{kg}$ cada 3 semanas $^{9}$. En un estudio publicado recientemente que incluyó a 3,647 pacientes que fueron tratados en 10 diferentes ensayos clínicos, la mediana de peso fue de $77.2 \mathrm{~kg}$ y se concluyó que no había diferencias en parámetros farmacocinéticos entre la dosis de $2 \mathrm{mg} / \mathrm{kg}$ cada 3 semanas vs. la dosis fija de 200 mg cada 3 semanas (Fig. 3) ${ }^{10}$. Con el estudio anterior se estableció que las posibles ventajas de la dosis fija son: una preparación más fácil y rápida, con menos porcentaje de errores en la preparación, ya que se disponen de viales de $100 \mathrm{mg}$.

Sin embargo, una limitante con este tipo de tratamientos es el elevado costo, que no permite que todos los pacientes candidatos a recibirlas puedan hacerlo. Por otra parte, el empleo de dosis fijas habitualmente lleva a un exceso de dosis del fármaco administrado, resultando en un gasto excesivo que en un sistema de salud como el mexicano no es factible. Además, la llegada de estos dos fármacos a los sistemas de salud 
Tabla 3. Exceso de costos del tratamiento del cáncer de pulmón de células no pequeñas (CPCNP) con nivolumab por 1 año de tratamiento con esquema de dosis fija

\begin{tabular}{|c|c|c|c|c|c|c|c|}
\hline Peso & Peso (kg) & $\begin{array}{c}\text { Dosis/kg } \\
\text { nivolumab } \\
\text { (promedio) }\end{array}$ & $\begin{array}{l}\text { Exceso de } \\
\text { dosis } \\
\text { nivolumab }\end{array}$ & $\begin{array}{l}\text { Excesos de } \\
\text { costos } \\
\text { nivolumab }\end{array}$ & $\begin{array}{l}\text { Costo anual } \\
\text { nivolumab } \\
\text { dosis fija }\end{array}$ & $\begin{array}{l}\text { Costo anual } \\
\text { nivolumab } \\
\text { dosis/kg }\end{array}$ & $\begin{array}{l}\text { Exceso de gasto } \\
\text { anual por } \\
\text { paciente }\end{array}$ \\
\hline Mínimo & 34.5 & $103.5 \mathrm{mg}$ & +136.5 mg & $+\$ 46,683$ & $\$ 2,134,080.00$ & $\$ 1,213,758.00$ & $\$ 920,322$ \\
\hline Máximo & 130.8 & $392.4 \mathrm{mg}$ & $-152.4 \mathrm{mg}$ & $-\$ 52,150$ & $\$ 2,134,080.00$ & & $-\$ 1,355,900$ \\
\hline $\begin{array}{l}\text { Mediana } \\
\text { (DE) }\end{array}$ & $\begin{array}{c}65 \\
13.11\end{array}$ & $195 \mathrm{mg}$ & $45 \mathrm{mg}$ & $+\$ 15,390$ & $\$ 2,134,080.00$ & $\$ 1,733,940$ & $\$ 400,140$ \\
\hline$\leq 50 \mathrm{~kg}$ & $\begin{array}{l}100 / 792 \\
(12.5 \%)\end{array}$ & $150 \mathrm{mg}$ & $90 \mathrm{mg}$ & $+\$ 30,780$ & $\$ 2,134,080.00$ & $\$ 1,333,800$ & $\$ 800.280$ \\
\hline$\geq 51-60 \mathrm{~kg}$ & $\begin{array}{l}199 / 792 \\
(25.1 \%)\end{array}$ & $165 \mathrm{mg}$ & $75 \mathrm{mg}$ & $+\$ 25,650$ & $\$ 2,134,080.00$ & $\$ 1,467,180$ & $\$ 666,900$ \\
\hline$\geq 61-70 \mathrm{~kg}$ & $\begin{array}{l}251 / 792 \\
(31.7 \%)\end{array}$ & $195 \mathrm{mg}$ & $45 \mathrm{mg}$ & $+\$ 15,390$ & $\$ 2,134,080.00$ & $\$ 1,733,940$ & $\$ 400,140$ \\
\hline$\geq 71-80 \mathrm{~kg}$ & $\begin{array}{c}150 / 792 \\
(19 \%)\end{array}$ & $225 \mathrm{mg}$ & $15 \mathrm{mg}$ & $+\$ 5,130$ & $\$ 2,134,080.00$ & $\$ 2,000,700$ & $\$ 133,380$ \\
\hline$\geq 81-90 \mathrm{~kg}$ & $\begin{array}{l}60 / 792 \\
(7.6 \%)\end{array}$ & $255 \mathrm{mg}$ & $-15 \mathrm{mg}$ & $-\$ 5,130$ & $\$ 2,134,080.00$ & $\$ 2,267,460$ & $-\$ 133,380$ \\
\hline$\geq 91-100 \mathrm{~kg}$ & $\begin{array}{c}24 / 792 \\
(3 \%)\end{array}$ & $285 \mathrm{mg}$ & $-45 \mathrm{mg}$ & $-\$ 15,390$ & $\$ 2,134,080.00$ & $\$ 2,534,220$ & $-\$ 400,140$ \\
\hline$\geq 101 \mathrm{~kg}$ & $\begin{array}{l}8 / 792 \\
(1 \%)\end{array}$ & $303 \mathrm{mg}$ & $-90 \mathrm{mg}$ & $-\$ 30,780$ & $\$ 2,134,080.00$ & $\$ 2,934,360$ & $-\$ 800,280$ \\
\hline
\end{tabular}

Indicaciones de nivolumab:

I. 2. 'ínea de tratamiento como monoterapia en CPCNP escamoso (cualquier valor de PD-L1).

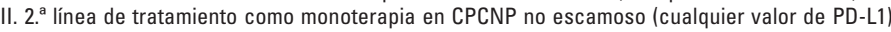

Resultados en los estudios pivotales:

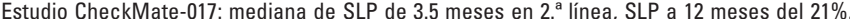

Estudio CheckMate-057: mediana de SLP de 2.3 meses en 2. ${ }^{a}$ línea, SLP a 12 meses del $19 \%$.

Costo de $240 \mathrm{mg}$ de nivolumab cada 2 semanas $=\$ 82,080.00$

PD-L1: programmed death ligand 1; SLP: Supervivencia Libre de Progresión

de México que más derechohabientes tienen (Instituto Mexicano del Seguro Social [IMSS] e Instituto de Seguridad y Servicios Sociales de los Trabajadores del Estado [ISSSTE]) representa todo un reto por el elevado costo de estos medicamentos. En un trabajo realizado por nuestro grupo y enviado recientemente al Congreso mundial de cáncer de pulmón edición 2019 (WLCL 2019), nos dimos a la tarea de analizar el peso de 792 pacientes consecutivos atendidos en la unidad funcional de tumores torácicos durante el periodo 2016-2018, candidatos a recibir tratamiento con inmunoterapia en primera o segunda línea. La mediana de peso fue de $65 \mathrm{~kg}$ (desviación estándar [DE]: $\pm 13.2 \mathrm{~kg}$ ). Se determinó que el costo por cada mg de pembrolizumab es de $\$ 750.00$ y el costo de cada mg de nivolumab es de $\$ 342.00$ (Tabla 1).

Basados en la información anterior, por cada paciente tratado durante un año con la dosis fija de pembrolizumab (200 mg cada 3 semanas), el exceso de costos del tratamiento sería de $\$ 892,500.00$, lo cual multiplicado por 100 pacientes daría un gasto de $\$ 89,250,000$, lo cual alcanzaría para cubrir el tratamiento de otros 54 pacientes durante un año si se utiliza el esquema de tratamiento basado en $2 \mathrm{mg} / \mathrm{kg}$ cada 3 semanas (Tabla 2). En cuanto al nivolumab, con el esquema de dosis fija (240 mg cada 2 semanas), por cada paciente tratado durante un año el exceso de gasto es de $\$ 400,140.00$, y por cada 100 pacientes tratados durante un año con la dosis fija, el exceso de gasto sería de $\$ 40,014,000$. Este gasto excesivo alcanzaría para tratar a otros 23 pacientes si utilizáramos el esquema de $3 \mathrm{mg} / \mathrm{kg}$ cada 3 semanas (Tabla 3 ).

El cálculo de la dosis de los tratamientos oncológicos, particularmente con quimioterapia, siempre ha sido con base en la superficie corporal (calculada a partir del peso y talla del paciente) 0 a los kilogramos de peso del paciente. Con lo anterior, se busca reducir la toxicidad de los tratamientos y optimizar la dosis de quimioterapia empleada. Para ello se dispone de presentaciones de tratamiento adecuados para la correcta 
preparación del tratamiento. El personal que administra este tipo de tratamientos cuenta con una gran experiencia y supervisión que evita los errores al momento de la aplicación. Los fundamentos anteriores también deberían aplicar a las inmunoterapias, por lo que deberíamos exigir presentaciones de medicamentos adecuadas para el cálculo de la dosis tomando en cuenta el peso del paciente y emplear la dosis calculada por kilogramo de peso como fueron aprobadas inicialmente, lo cual nos permitiría tratar a más pacientes con la misma cantidad de dinero.

¿Estamos preparados para migrar al esquema de dosis fija de nivolumab y pembrolizumab? La respuesta es un rotundo no. No lo estamos, sobre todo en el tema económico. Como sociedad debemos optimizar recursos y tratar a más pacientes con el mismo presupuesto, por lo que los esquemas con dosis calculadas por kilogramo de peso deberían ser las que utilicemos para nuestros pacientes y sería adecuado exigir presentaciones adecuadas de ellos que nos permitan calcular la dosis que cada paciente requiere, evitando el gasto excesivo y beneficiando a más pacientes.

\section{Bibliografía}

1. Topalian SL, Hodi FS, Brahmer JR, Gettinger SN, Smith DC, McDermott DF, et al. Safety, activity, and immune correlates of anti-PD-1 antibody in cancer. N Engl J Med. 2012;366:2443-54.

2. Brahmer J, Reckamp KL, Baas P, Crinò L, Eberhardt WE, Poddubskaya E, et al. Nivolumab versus docetaxel in advanced squamous-cell non-smaII-cell lung cancer. N Engl J Med. 2015;373:123-35.

3. Borghaei H, Paz-Ares L, Horn L, Spigel DR, Steins M, Ready NE, et al. Nivolumab versus docetaxel in advanced nonsquamous non-small-cell lung cancer. N Engl J Med. 2015;373:1627-39.

4. Bajaj G, Wang X, Agrawal S, Gupta M, Roy A, Feng Y. Model-based population pharmacokinetic analysis of nivolumab in patients with solid tumors. CPT Pharmacometrics Syst Pharmacol. 2017;6:58-66.

5. Zhao X, Suryawanshi S, Hruska M, Feng Y, Wang X, Shen J, et al. Assessment of nivolumab benefit-risk profile of a $240-\mathrm{mg}$ flat dose relative to a $3-\mathrm{mg} / \mathrm{kg}$ dosing regimen in patients with advanced tumors. Ann Oncol. 2017;28(8):2002-8.

6. Patnaik A, Kang SP, Rasco D. et al. Phase I study of pembrolizumab (MK-3475; anti-PD-1 monoclonal antibody) in patients with advanced solid tumors. Clin Cancer Res. 2015;21(19):4286-93.

7. Kang SP, Gergich K, Lubiniecki GM, de Alwis DP, Chen C, Tice MAB, et al. Pembrolizumab KEYNOTE-001: an adaptive study leading to accelerated approval for two indications and a companion diagnostic. Ann Oncol. 2017;28(6):1388-98.

8. Chatterjee M, Turner DC, Felip E, Lena H, Cappuzzo F, Horn L, et al. Systematic evaluation of pembrolizumab dosing in patients with advanced non-small-cell lung cancer. Ann Oncol. 2016;27(7):1291-8.

9. Herbst RS, Baas P, Kim DW, Felip E, Pérez-Gracia JL, Han JY, et al. Pembrolizumab versus docetaxel for previously treated, PD-L1-positive, advanced non-small-cell lung cancer (KEYNOTE-010): a randomised controlled trial. Lancet. 2016;387:1540-50.

10. Freshwater T, Kondic A, Ahamadi M, Li CH, de Greef R, de Alwis D, et al. Evaluation of dosing strategy for pembrolizumab for oncology indications. J Immunother Cancer. 2017;5:43. 